\title{
Revised SCLP-simplex Algorithm with Application to Large-Scale Fluid Processing Networks
}

\author{
Evgeny Shindin, Michael Masin, Gideon Weiss and Alexander Zadorojniy
}

\begin{abstract}
We describe an efficient implementation of a recent simplex-type algorithm for the exact solution of separated continuous linear programs, and compare it with linear programming approximation of these problems obtained via discretization of the time horizon. The implementation overcomes many numerical pitfalls often neglected in theoretical analysis allowing better accuracy or acceleration up to several orders of magnitude both versus previous implementation of the simplex-type algorithms and versus a state-of-the-art LP solver using discretization. Numerical study includes medium, large, and very large examples of scheduling problems and problems of control of fluid processing networks. We discuss online and offline optimization settings for various applications and outline future research directions.
\end{abstract}

\section{INTRODUCTION}

In this paper we present an implementation and evaluate the performance of a simplex-type algorithm for the solution of a separated continuous linear programming problem (SCLP):

$$
\begin{array}{ll}
\max _{u(t), x(t)} & \int_{0}^{T}\left(\gamma^{\top}+(T-t) c^{\top}\right) u(t)+d^{\top} x(t) d t, \\
\text { s.t. } & \int_{0}^{t} G u(s) d s+F x(t) \leq \alpha+a t, \\
& H u(t) \leq b, \\
& x(t), u(t) \geq 0, \quad 0 \leq t \leq T,
\end{array}
$$

SCLP problems are a special case of continuous linear programs (CLP) formulated by Bellman [1], and were first suggested for the solution of job shop scheduling problems by Anderson [2]. Many important problems can be formulated as SCLP's, but up to date these problems were always solved by discretizing the time. The simplex-type algorithm (SCLP-simplex) studied here was derived by Weiss [3], and in this paper we present the first streamlined stable and efficient implementation of this algorithm and compare its performance to the discretized LP approximation.

SCLP-simplex has several important advantages:

Research of G. Weiss funded by ISF Grants 249/02, 454/05, 711/09 and 286/13. Research of M. Masin, E. Shindin and A Zadorojniy funded by the EU Commission's H2020 Program under grant agreements No 732105 and No 780788 .

E. Shindin is with IBM Research - Haifa, Mount Carmel, Haifa, 3498825, Israel evgensh@il.ibm.com

M. Masin is with Optibus, Eder 48a, Haifa, 3475293, Israel michael.masin@optibus.com

G. Weiss is with Department of Statistics, University of Haifa Mount Carmel, Haifa, 3498838, Israel gweiss@stat.haifa. ac.il

A. Zadorojniy is with IBM Research - Haifa, Mount Carmel, Haifa, 3498825, Israel zalex@il.ibm.com
- SCLP shares many of the properties of standard LP: it has a symmetric dual, it satisfies strong duality, solutions are obtained at extreme points, and extreme points are characterized by a combinatorial analog of basic solutions, with a well defined pivot operation.

- SCLP-simplex solves SCLP in a finite number of iterations, exactly, using a parametric approach similar to Lemke's algorithm [4].

- The form of the SCLP-simplex solution lends itself to perform sensitivity analysis.

- The SCLP-simplex can be implemented as a model predictive control for online long term optimization.

These can be contrasted to some disadvantages of time discretization:

- The time discretized LP are large and may require substantial computational effort.

- The solution of the discretized approximation of SCLP may be inaccurate. To be accurate some regions of the time horizon need only rough discretization, while others require a very fine discretization. However it is difficult to tell where these regions are, so the quality of the approximation is very uncertain.

- The structure of the optimal solution is lost in the discretized solution with many spurious basic variables that reduce the value of sensitivity analysis, and make it practically unsuitable for model predictive control, where a new discretized problem needs to be solved from scratch in every update.

Despite the advantages of the SCLP-simplex of [3] it has so far only been implemented by the author as a trial pilot aimed to verify the algorithm on very small examples. While it received many citations referring to the theoretical results, it has never been used in practice.

Our contribution in this paper is a revised SCLPsimplex, that is taking advantage of several computational techniques, and is the first stable and efficient implementation of the algorithm. As a result, the revised SCLP-simplex often outperforms the discretized method, both in computation time as well as solution quality: for some large problems the discretization method fails to complete the calculation with reasonable accuracy, while SCLP-simplex solves these problems in a reasonable time, with perfect accuracy.

The rest of this paper is structured as follows: Section II provides the structure of optimal solutions and briefly describes the SCLP-simplex algorithm. Section III discusses details of our revised implementation. Section IV]describes the potential applications and sets the experimental setup, 
followed by a computational study in Section V Section VI summarizes the work and discusses future directions.

\section{BACKGROUND ON SCLP}

The main relevant SCLP references to our approach are [1], [2], [5], [6], [7], [8], [9]. Additional approaches are shown in [10], [11], [12]. The SCLP-simplex algorithm is based on [3], several extensions and generalizations are shown in [13], [14], [15], [16], [17]. In this section we describe the structure of optimal solutions

In (1), the matrices $G, H, F$ are $K \times J, I \times J$, $K \times L$ dimensional respectively, and we number the slacks $x_{1}, \ldots, x_{K}$, and $u_{J+1}, \ldots, u_{J+I}$. Denote by $\mathbf{K}=$ $(1, \ldots, K+L)$ the indexes of the primal state variables $x_{k}(t)$ and by $\mathbf{J}=(1, \ldots, J+I)$ the indexes of the primal control variables $u_{j}(t)$. The symmetric dual to $\sqrt{1}$ is

$$
\begin{array}{ll}
\min _{p(t), q(t)} & \int_{0}^{T}\left(\alpha^{\top}+(T-t) a^{\top}\right) p(t)+b^{\top} q(t) d t, \\
\text { s.t. } & \int_{0}^{t} G^{\top} p(s) d s+H^{\top} q(t) \geq \gamma+c t, \\
& F^{\top} p(t) \geq d, \\
& q(t), p(t) \geq 0, \quad 0 \leq t \leq T,
\end{array}
$$

with dual state variables, including slacks, $q_{j}(t), j \in \mathbf{J}$ and dual control variables $p_{k}(t), k \in \mathbf{K}$. Note that the dual problem runs in reversed time. Complementary slackness is defined by:

$$
\int_{0}^{T} x(t)^{\top} p(T-t) d t=\int_{0}^{T} u(t)^{\top} q(T-t) d t=0
$$

Under easily checked feasibility and boundedness conditions, and under non-degeneracy, SCLP has a unique strongly dual solution. The optimal solution has piecewise constant primal and dual controls and continuous piecewise linear primal and dual state variables, with breakpoints $0=t_{0}<t_{1}<\cdots<t_{N}=T$. The solution is then fully described by the breakpoints, by the initial state values $x(0)=x^{0}, q(0)=q^{N}$, and by the values of the controls and of the derivatives of the states $u_{j}^{n}=u_{j}(t), p_{k}^{n}=p_{k}(T-t), \dot{x}_{k}^{n}=\dot{x}_{k}(t), \dot{q}_{j}^{n}=\dot{q}_{j}(T-t)$ for $t_{n-1}<t<t_{n}, n=1, \ldots, N$. The values of the primal and dual states at the breakpoints are $x_{k}^{n}=x_{k}\left(t_{n}\right), q_{j}^{n}=$ $q_{j}\left(T-t_{n}\right), n=0, \ldots, N$. The initial values, $x^{0}, q^{N}$, are optimal solutions of the Boundary-LP:

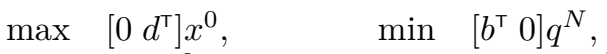

$$
\begin{aligned}
& \begin{array}{lc}
\text { s.t. } & {[I F] x^{0}=\alpha, \quad \text { s.t. } \quad\left[H^{\top}-I\right] q^{N}=\gamma,} \\
& x^{0} \geq 0,
\end{array}
\end{aligned}
$$

with $\mathcal{K}_{0}, \mathcal{J}_{N+1}$ the indexes of the basic variables $x_{k}^{0}, q_{j}^{N}$. Note: the LP for $x^{0}, q^{N}$ does not involve $T$, so the boundary values are the same for all time horizons.

Values of the controls and slopes of states in the intervals are complementary slack basic solutions of the primal and dual Rates-LP(K, $\mathcal{J})$ :

$$
\begin{array}{lll}
\max & {\left[c^{\top} 0\right] u+\left[\begin{array}{ll}
0 & d^{\top}
\end{array}\right] \dot{x}} & \dot{x}_{k} \in \mathbb{R} \forall k \in \mathcal{K}, \\
\text { s.t. } & {\left[\begin{array}{ll}
G & 0
\end{array}\right] u+\left[\begin{array}{ll}
I & F
\end{array}\right] \dot{x}=a,} & \dot{x}_{k} \in \mathbb{R}^{+} \forall k \notin \mathcal{K}, \\
& {\left[\begin{array}{lll}
H & I
\end{array}\right] u \quad u_{j}=0 \forall j \in \mathcal{J},} \\
& & u_{j} \in \mathbb{R}^{+} \forall j \notin \mathcal{J},
\end{array}
$$

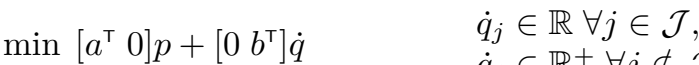

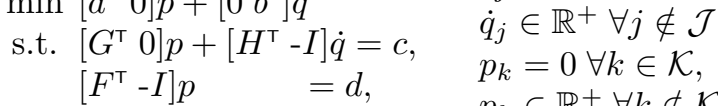

$$
\begin{aligned}
& p_{k} \in \mathbb{R}^{+} \forall k \notin \mathcal{K},
\end{aligned}
$$

where for interval $\left(t_{n-1}, t_{n}\right)$ the primal basis is $B_{n}=$ $\left\{u_{j}^{n}, \dot{x}_{k}^{n}: j \notin \mathcal{J}_{n}, k \in \mathcal{K}_{n}\right\}$ with complementary dual basis $B_{n}^{*}=\left\{p_{k}^{n}, \dot{q}_{j}^{n}: k \notin \mathcal{K}_{n}, j \in \mathcal{J}_{n}\right\}$.

The bases have the following properties:

- Compatibility to the boundary: $\mathcal{K}_{0} \subseteq \mathcal{K}_{1}, \mathcal{J}_{N+1} \subseteq \mathcal{J}_{N}$. - Adjacency: $B_{n}, B_{n+1}$ are adjacent: in the pivot $B_{n} \rightarrow$ $B_{n+1}$ a single basic variable $v^{n}$ leaves the basis and a single basic variable $w^{n}$ enters.

The breakpoints $t_{1}, \ldots, t_{N-1}$ are determined by the following equations for the interval lengths $\tau_{n}=t_{n}-t_{n-1}$ :

$$
\begin{aligned}
& x_{k}\left(t_{n}\right)=x_{k}^{0}+\sum_{m=1}^{n} \dot{x}_{k}^{m} \tau_{m}=0 \quad \text { if } v^{n}=\dot{x}_{k}, \\
& q_{j}\left(T-t_{n}\right)=q_{j}^{N}+\sum_{m=N}^{n+1} \dot{q}_{j}^{m} \tau_{m}=0 \text { if } v^{n}=u_{j}, \\
& \tau_{1}+\cdots+\tau_{N}=T .
\end{aligned}
$$

The remaining values are determined by:

$$
\begin{aligned}
& x_{k}\left(t_{n}\right)=x_{k}^{0}+\sum_{m=1}^{n} \dot{x}_{k}^{m} \tau_{m}, \\
& q_{j}\left(T-t_{n}\right)=q_{j}^{N}+\sum_{m=N}^{n+1} \dot{q}_{j}^{m} \tau_{m} .
\end{aligned}
$$

Given a sequence of adjacent bases $B_{1}, \ldots, B_{N}$ we can calculate all the controls and slopes of states, the breakpoints, and the values of the primal and dual states at all breakpoints. It is an optimal base sequence if:

Theorem 2.1 ([3]): If a sequence of bases $B_{1}, \ldots, B_{N}$ are compatible with $\mathcal{K}_{0}, \mathcal{J}_{N+1}$ and are adjacent, and if all the values of the primal and dual state variables and the interval lengths determined by equations (4)-(8) are positive, then this is an optimal solution of the SCLP.

The SCLP-simplex algorithm is similar to the parametric self dual simplex algorithm, also known as Lemke's [4] algorithm, for the solution of standard LP. In Lemke's algorithm a pair of dual LP's is solved parametrically, starting from an objective of -1 's and a r.h.s. of 1's with the trivial optimal solution where the primal and dual basic variables are the slacks. Then it solves all the LP's along the parametric line $\mathcal{L}(\theta)=(1-\theta)\left[\begin{array}{c}-1 \\ 1\end{array}\right]+\theta\left[\begin{array}{l}c \\ b\end{array}\right]$. The solution partitions $0<\theta_{1}<\cdots<\theta_{M}=1$, and at each $\theta_{\ell}$ either a primal or a dual variable shrinks to 0 , and a single pivot or several pivots are performed to obtain the optimal basis for $\theta>\theta_{\ell}$.

The SCLP-simplex is initiated by solving (4) for $x(0), q(0)$ and obtaining $B_{1}$, the optimal basis for Rates$\operatorname{LP}\left(\mathcal{K}_{0}, \mathcal{J}_{N+1}\right) . B_{1}$ is the initial optimal base sequence for small time horizons. Then, in analogy to Lemke's algorithm, SCLP-simplex solves SCLP parametrically, by increasing the time horizon $\theta T$ over $0<\theta \leq 1$, with iterations needed at $0<\theta_{1}<\cdots<\theta_{M}=1$. In each validity range $\theta_{\ell-1}<\theta<\theta_{\ell}$, the optimal solution is defined by an optimal base sequence $B_{1}, \ldots, B_{N}$, and the values of $x^{n}, q^{n}, \tau_{n}$ are affine functions of $\theta$ with well defined derivatives $\delta(\cdot)=\frac{d(\cdot)}{d \theta}$. At $\theta_{\ell}$ a collision occurs: either one or several intervals or else a primal or a dual state variable shrink to zero at a breakpoint $t_{n}$, and an SCLP-pivot is performed to obtain the optimal base 
sequence in the next validity range.

The main difference is that in Lemke's LP at each step, only the one basis defines the optimal solution, while in SCLP-simplex the optimal solution consists of the base sequence. Each iteration of SCLP-simplex consists of two steps: calculation of the validity range, and SCLP-pivot.

- Calculation of the validity range, $\theta_{\ell}$

$$
\theta_{\ell}=\theta_{\ell-1}+\inf _{\delta(\cdot)<0}\left\{\theta:-\frac{\tau_{n}}{\delta \tau_{n}},-\frac{x_{k}^{n}}{\delta x_{k}^{n}},-\frac{q_{j}^{n}}{\delta q_{j}^{n}}\right\} .
$$

We then obtain the type of collision, interval shrinking or state variable shrinking, the location of the collision, $t_{n}$, and the bases on both sides to the collision, $B^{\prime}, B^{\prime \prime}$.

- SCLP-pivot, consists of the the following:

- If intervals shrunk to 0 , remove bases between $B^{\prime}, B^{\prime \prime}$. If $B^{\prime}, B^{\prime \prime}$ are adjacent, you found the new base sequence. - Otherwise, $B^{\prime} \backslash B^{\prime \prime}=\left\{v_{1}, v_{2}\right\}$, choose proper $v^{\prime}, v^{\prime \prime} \in$ $\left\{v_{1}, v_{2}\right\}$, and solve Rates-LP $\left(\mathcal{K}^{*}, \mathcal{J}^{*}\right)$, where $\mathcal{K}^{*}=\{k$ : $\left.\dot{x}_{k} \in B^{\prime}\right\} \backslash v^{\prime \prime}, \mathcal{J}^{*}=\left\{j: u_{j} \notin B^{\prime \prime}\right\} \cup v^{\prime}$ to obtain basis $D$. If $D$ is adjacent to $B^{\prime}, B^{\prime \prime}$, insert it between $B^{\prime}, B^{\prime \prime}$, you found the new base sequence.

- Otherwise, formulate a subproblem, which is an SCLP of smaller dimension, and solve it to obtain an optimal base sequence $D_{1}, \ldots, D_{L}$, and insert it between $B^{\prime}, B^{\prime \prime}$, you found the new base sequence.

For collisions at 0 or $T$ the steps of the pivot are slightly modified.

\section{Revised SCLP-Simplex Algorithm}

SCLP-simplex [3] will always work perfectly under the following conditions: The problem needs to be nondegenerate, and all calculations need to be done with perfect accuracy. However, previous implementation, that was intended only as a pilot for concept verification, used Matlab with floating point calculations and was vulnerable to degeneracy and inaccuracies. Moreover straightforward implementation suffered from memory and performance issues, that substantially slowed down the algorithm. As a result only problems with $K+L+$ $J+I \leq 100$ could be solved before the program crashed or ran out of time.

To improve performance and numerical stability of the SCLP-simplex algorithm we thoroughly analyzed each step and developed the revised SCLP-simplex algorithm. Python implementation of the algorithm is available at GitHub https://github.com/IBM/SCLPsolver.

The following analysis and implementation enhancements led to substantial performance gains.

- Base sequence representation is one of the problematic points of the SCLP-simplex: the choice to store only the indexes of the basic variables, and re-solve all Rates-LP fresh at each iteration requires an impractical amount of computations, and storage of the simplex dictionaries for all bases involves memory issues, in storage and in updating. Since all bases are adjacent our algorithm stores simplex dictionaries only for some of them, keeping the list of pivots between all bases. This requires more computations when a new basis
$(D)$ needs to be calculated, but drastically reduces the required memory. The code obtains the available RAM and adjusts the number of stored dictionaries accordingly, maintaining evenly spaced dictionaries.

- Values of all $\dot{x}, \dot{q}$ are kept since only a small part of them is updated during the SCLP pivot.

- Equations (7) are re-structured to increase sparsity. The resulting system is solved for $\delta \tau\left(\theta_{\ell}\right)$ using $\mathrm{LU}$ factorization, while $\tau\left(\theta_{\ell}\right)$ is calculated as $\tau\left(\theta_{\ell-1}\right)+$ $\delta \tau\left(\theta_{\ell-1}\right)$. In a case when exactly one interval shrinks to 0 and is replaced by a single interval, the corresponding SCLP pivot changes only one column of coefficients in (7). In this case we use product form of inverse (PFI) to calculate $\delta \tau\left(\theta_{\ell}\right)$ from $\delta \tau\left(\theta_{\ell-1)}\right.$ using $\mathrm{LU}$ decomposition obtained in the previous iteration. Such design reduces the number of operations from $\mathcal{O}\left(N^{3}\right)$ to $\mathcal{O}(N)$ complexity.

- To evaluate $\theta_{\ell}-\theta_{\ell-1}$ in $(9)$ we use only the values of $\delta \tau_{n}, \tau_{n}$ and of $x^{n}, q^{n}$ at local minima where $\delta x^{n}, \delta q^{n}$ are negative. This allows us to solve only part of 8 . For this purpose we keep a list of all the local minima of $x(t), q(t)$. Updating this list involves only small changes in each iteration.

- The basis $D$ is calculated by pivoting the simplex dictionary of $B^{\prime}$ or $B^{\prime \prime}$ using non-standard pivoting rules, where entering and leaving variables are determined by $B^{\prime \prime} \backslash B^{\prime}$ and $B^{\prime} \backslash B^{\prime \prime}$ and by the type of the collision. If and optimal $D$ which is adjacent to both $B^{\prime}, B^{\prime \prime}$ exists, it is always found by this single pivot.

- To calculate $B^{\prime \prime} \backslash B^{\prime}$ and $B^{\prime} \backslash B^{\prime \prime}$ we use the list of pivots, and avoid computationally expensive set differences.

- The subproblems are solved with reduced dimension, and are therefore quite small.

Numerical pitfalls lead to poor stability of the naive implementation that depends on non-degeneracy of all dictionaries, and on correct identification of the collision types. In theory, perturbation of the data, in particular of $a$ and $c$ can achieve all the required non-degeneracy, and ensure unique execution of all the steps of the algorithm. However, in practice, accumulation of numerical errors due to floating point operations may impede such clean runs. In most cases numerical problems arise when we should decide if a value is 0 or just a small floating point number or when we should compare close floating point numbers to determine the sequence of intervals that shrink to 0 . Numerical instabilities lead to incorrect collision classification which may not be recognized at the classification time, but will appear in further steps. This creates situations that are impossible in theory, but do occur in practice, such as

- Incorrect collision: $\tau_{n}, \ldots, \tau_{n^{\prime}}$ shrink to 0 in the middle of the base sequence, but $\left|B_{n-1} \backslash B_{n^{\prime}+1}\right|>2$.

- Incorrect pivot: the new basis $D$ is adjacent to $B^{\prime}$ and $B^{\prime \prime}$ but values of $\dot{x}_{k}$ and/or $\dot{q}_{j}$ for this basis leading to a discontinuity in the state variables.

- Incorrect subproblem formulation: during the solution of a subproblem the base sequence $D_{1}, \ldots, D_{L}$ arrives at 
an infeasible or unbounded basis $D_{l}$, or the subproblem parametric line reaches $\theta=1$, but $D_{1}$ and $B^{\prime}$ or $D_{L}$ and $B^{\prime \prime}$ are not adjacent.

- Zero lengths interval shrink: a new interval obtained at the previous iteration shrinks, introducing infinite loop, where the parametric line is not moving forward, or a number of zero lengths intervals shrink, which impedes the collision classification.

In these cases we return to the classification step, then change the numerical tolerances and reclassify the collision. Once the problem is resolved, tolerances are readjusted. In addition, starting from an iteration where the collision classification was not clear, we store information that allows us to go back. If the reclassification fails, we go to an earlier iteration trying to reclassify the collision there.

\section{Applications and Experimental Setup}

The original motivation for SCLP was to define tractable optimization models for the job shop scheduling problems [2]. For example, micro-chips wafer fabricalion: starting as a wafer of pure silicon crystal, the wafer undergoes up to 1200 operations revisiting a set of up to 60 workstations, in a re-entrant line production process, to produce several hundred computer chips, in a cycle time of some six weeks. The problem is to control the movement of some 60,000 wafers over a time horizon of 6 weeks, with work in process value of $200 \times 10^{6} \$$. A rich literature on this problem includes [18], [19], [20], [21]. Specifically, solution of SCLP to these problems is described in [10], [22] with small to medium size examples. This application motivates our first experiment solving problems of the full size.

- Transient control of multi-class queueing networks: Items of several types arrive at the system, and need to follow individual paths through various service stations, and we need to control admissions, routing and sequencing items throughout the system [23], [24], [25], [26], [27], [28]. In the second experiment we test the revised SCLPalgorithm to solve the SCLP formulation of this problem enabling asymptotically optimal control of the stochastic system [29].

[22] have shown that robust optimization of both problems can also be formulated as SCLP. The formulation increases the problem sizes even further. Moreover, many additional application benefit from the proposed algorithm, e.g., health systems, where the flow of patients through emergency rooms, hospitalization, operating theaters, requires concerted use of resources, and patients follow complex paths through the system, see [30], [31]; the quickest evacuation problem for evacuation of stadiums, convention centers, amusement parks; and even the standard maximum flow problem over time usually solved via discretization of time can be easily solved as an SCLP.

We chose two types of SCLP problems to evaluate the performance of our revised SCLP-simplex: SCLP for a re-entrant line that approximates the wafer fab job shop scheduling problem, and SCLP for transient control of a multi-class queueing network, that approximates a stochastic service system.

The re-entrant line $S C L P$ : The state variable $x_{k}(t)$ is the quantity of wafers waiting to complete production step $k$, also referred to as contents of buffer $k$. The control variable $u_{k}(t)$ is the processing capacity allocated to buffer $k$. The objective is:

$$
\min _{u(t), x(t)} V=\int_{0}^{T}\left(h^{\top} x(t)+g^{\top} u(t)\right) d t
$$

where $h$ is the vector of holding costs, and $g$ the vector of operating costs. $G=\left[\begin{array}{ccccc}1 & 0 & 0 & \ldots & 0 \\ -1 & 1 & 0 & \ldots & 0 \\ 0 & -1 & 1 & \ldots & 0 \\ \vdots & \ddots & \ddots & \ddots & \vdots \\ 0 & \ldots & 0 & -1 & 1\end{array}\right]$ represents the reentrant line, with items moving from buffer 1 to 2 etc, until $K$ and out. $H$ is the resource constraints matrix where rows $i=1, \ldots, I$ represent workstations, and $H_{i, k}=m_{k}$ where $m_{k}$ is the processing time per item at production step $k$ performed at workstation $i$. The vector $b$ is all 1's, as capacity of workstation $i$. Initial inventory in buffer $k$ is $\alpha_{k}, a_{k}$ is the rate of wafers input to buffer $k$, often $a_{1}>0$, and all other $a_{k}=0$. To conform to (1) we substitute $x(t)=\alpha+a t-\int_{0}^{t} G u(s) d s$ in the objective to obtain a surrogate equivalent maximization objective, where $\gamma=-g$ and $c=h^{\top} G$. For this problem $F$ and $d$ of (1) are nil.

Transient control MCQN: queues $k=1, \ldots, K$ have $x_{k}(t)$ discrete items waiting for processing. Activity $j=$ $1, \ldots, J$ will process an item from $k=k(j)$ for a random time with mean $m_{j}$, and then route the completed item to queue $l$ with probability $p_{k(j), l}^{j}$, or send it out of the system with probability $1-\sum_{l} p_{k(j), l}^{j}$. To do so it will use workstation $i=s(j)$. Items arrive at queue $k$ in a random stream, at rate $a_{k}$. We wish to decide which activities to employ at each time unit, so as to minimize the expected sojourn times or equivalently the (weighted) queue lengths, starting from some initial $x_{k}(0)$, over finite time horizon $[0, T]$. The SCLP approximation has

- $G$ is $K \times J$ with elements: $G_{l, j}=\left\{\begin{array}{ll}1, & l=k(j) \\ -p_{k(j), l}^{j}, & l \neq k(j)\end{array}\right.$.

- $H$ is $I \times J$ with elements $H_{i, j}=\left\{\begin{array}{ll}m_{j}, & s(j)=i \\ 0, & s(j) \neq i\end{array}\right.$.

\section{Computational Results}

We compare our implementation of SCLP-simplex vs. LP discretization with up to 1000 time intervals. for the two problem classes presented in Section IV] For each class we defined five settings with different amounts of servers, buffers, and job classes and randomly generated 10 problems. Randomly generated parameters include initial fluids, arrival rates, processing rates and time horizons. Their probability distributions, were chosen to avoid trivial or degenerate situation. All experiments were performed on Lenovo ThinkPad W541 notebook computer with Intel Core i7-4810MQ processor and 16GB RAM running Windows 10. SCLP-simplex ran on Python 3.7.7 with NumPy 1.18.1, linked to Intel MKL. Some vector 
and matrix operations parallelized using Cython and Intel OpenMP library. The discretized problems were solved by IBM Ilog Cplex Optimization Studio 12.10 using a barrier algorithm that showed the best performance for these problems. Both algorithms ran on eight cores.

\section{LP discretization}

Naive discretization may produce a quadratic number of unnecessary non-zero coefficients in the LP problem. Here we show a much more efficient discretization method to obtain an approximate solution of SCLP by regular LP solvers.

We consider a uniform time partition $0=t_{0}, \ldots, t_{N}=$ $T$, where $N$ is the number of intervals and for $n=$ $1, \ldots, N: t_{n}=t_{n-1}+\tau, \tau=T / N$. For each time interval $n=1, \ldots, N$ we define a vector of discrete controls $u[n]$ and state variables $x[n]$. Then the discretizition of problem (1) without $F$ and $d$ can be represented as:

$$
\begin{array}{ll}
\min _{u, x} & V_{L P}=\sum_{n=1}^{N}\left(\tau g^{\top} u[n]+0.5 h^{\top}(x[n]+x[n-1])\right. \\
\text { s.t. } & \tau G u[n]+x[n]-x[n-1]=a \tau \quad \forall n \\
& \text { with } \quad x[0]=\alpha \\
& H \cdot u[n] \leq b, \quad u[n], x[n] \geq 0, \quad \forall n .
\end{array}
$$

Results

The objective value $V$ provided by SCLP-simplex is the accurate theoretical minimum, the relative error is measured as $\left(V_{L P}-V\right) / V$. To compare performance, we measure relative time as the ratio of run times of CPLEX over SCLP-simplex. The results for re-entrant line and NCQN problems are shown in Table I] Fig. 1, 2 and

\begin{tabular}{|c|c|c|c|c|c|c|c|c|c|c|c|}
\hline \multirow{4}{*}{$\sum_{0}^{\infty}$} & \multirow{4}{*}{ 氖 } & \multirow{4}{*}{ 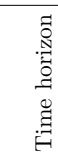 } & \multicolumn{3}{|c|}{ Average numbers } & \multicolumn{6}{|c|}{ Discretization } \\
\hline & & & \multirow{3}{*}{$\begin{array}{c}\text { Run } \\
\text { time, } \\
\text { sec }\end{array}$} & \multirow{3}{*}{ Steps } & \multirow{3}{*}{ 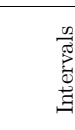 } & \multirow{2}{*}{\multicolumn{2}{|c|}{$\frac{10}{\text { Relative: }}$}} & \multirow{2}{*}{\multicolumn{2}{|c|}{$\begin{array}{c}100 \\
\text { Relative: }\end{array}$}} & \multicolumn{2}{|c|}{1000} \\
\hline & & & & & & & & & & Rel & ative: \\
\hline & & & & & & error & time & error & time & & \\
\hline 20 & 400 & 600 & 3.352 & 921.7 & 440.8 & 4.87 & & 0.400 & 0.763 & 0.022 & 12.449 \\
\hline 30 & 600 & 900 & 7.842 & 224 & 667.8 & 11.36 & & 1.013 & & 0.067 & \\
\hline 40 & 800 & 1200 & 16.570 & 3109.3 & 883.8 & 13.54 & & 1.236 & 0.214 & 0.090 & 4.4 \\
\hline 50 & 1000 & 1500 & 34.753 & 4402.3 & 1113.4 & 19.97 & 0.0099 & 1.866 & 0.125 & 0.146 & 2.429 \\
\hline 60 & 1200 & 1800 & 67.419 & 5699.0 & 1322.9 & 23.10 & 0.0066 & 2.183 & 0.081 & 0.176 & 1.617 \\
\hline
\end{tabular}
Table II] Fig. 3, 4, respectively.

\section{TABLE I}

RESults on RE-ENTRANT Line PROBLEMS

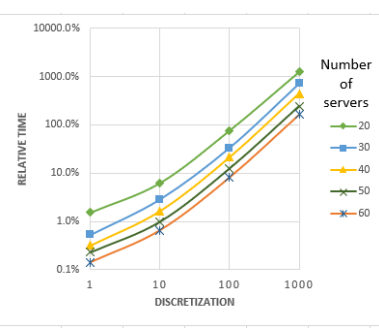

Fig. 1. Re-entrant line discretization relative time

For both problems, low discretization leads to nonoptimal solutions with large relative errors while high

Fig. 2. Re-entrant line discretization relative error discretization is resource-thirsty with long run times. We remark, that Cplex provide almost constant 100\% load on all processor cores, while for the revised SCLP-simplex implementation the load of all cores is not constant with $50 \%$ average load for all cores. This indicates that we may be able to further improve SCLP-simplex by exploiting

\begin{tabular}{|c|c|c|c|c|c|c|c|c|c|c|}
\hline \multirow{4}{*}{ 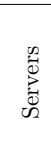 } & \multirow{4}{*}{ 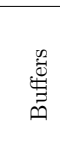 } & \multirow{4}{*}{ 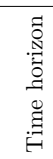 } & \multicolumn{3}{|c|}{ Average numbers } & \multicolumn{5}{|c|}{ Discretization } \\
\hline & & & \multirow{3}{*}{$\begin{array}{l}\text { Run } \\
\text { time, } \\
\text { sec }\end{array}$} & \multirow{3}{*}{ Steps } & \multirow{3}{*}{ 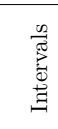 } & \multirow{2}{*}{$\begin{array}{c}10 \\
\text { Relative: }\end{array}$} & \multirow{2}{*}{\multicolumn{2}{|c|}{$\begin{array}{c}100 \\
\text { Relative: }\end{array}$}} & \multirow{2}{*}{\multicolumn{2}{|c|}{$\begin{array}{c}1000 \\
\text { Relative: }\end{array}$}} \\
\hline & & & & & & & & & & \\
\hline & & & & & & error time & error & time & & time \\
\hline 20 & 200 & 100 & 0.988 & & 271 & 1.2441 .027 & 0.049 & & & \\
\hline 40 & 400 & 100 & 4.81 & 188 & 535 & $\begin{array}{ll}93 & 1.3\end{array}$ & 0.034 & & 0.0009 & \\
\hline 60 & 600 & 100 & 17.098 & 3899 & 815 & 0.9131 .242 & 0.036 & 19.992 & 0.0009 & 946.591 \\
\hline 80 & 800 & 100 & 41.655 & 6424 & 1080 & $1.017 \quad 1.217$ & 0.039 & 23.967 & & \\
\hline 100 & 1000 & 100 & 91.809 & 9466 & 1356 & $0.922 \quad 1.051$ & 0.036 & 15.809 & & \\
\hline
\end{tabular}
more parallelization.

TABLE II

RESUlts ON MCQN PROBLEMS

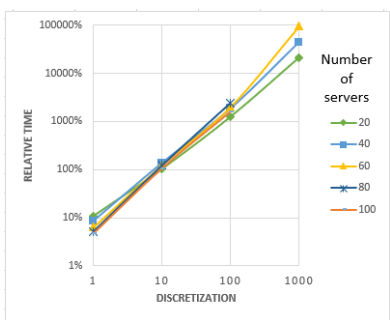

Fig. 3. MCQN discretization relative time

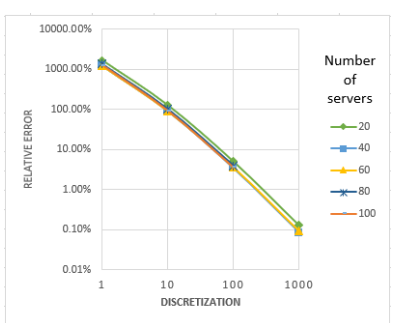

Fig. 4. MCQN discretization relative error

\section{Discussion}

To check our conjecture on linear empirical complexity similarly to Lemke's algorithm for LP, we compare the number of iterations $M$ (steps) to the problem dimensions that are usually expressed by the number of variables $(2 K)$ and the number of constraints $(K+I)$. Our results show that the number of steps is proportional to $2 K \cdot(K+I)$ and even decreasing with the problem size: the number of steps normalized by $2 K \cdot(K+I)$ is in a range of $[1.9 \ldots 3.3] \cdot 10^{-3}$ and $[4.3 \ldots 7.5] \cdot 10^{-3}$ for re-entrant line and MCQN problems, respectively, where the lower values happen for larger settings in both problem classes.

\section{Summary and Future Directions}

To summarize, we have shown that SCLP-simplex is viable for re-entrant line and control of queueing networks problems. It indicates that our implementation opens new opportunities in optimizing important classes of problems, as listed in Section IV. For all these applications we stress the important advantage of the SCLP-simplex in enabling us to do sensitivity analysis. In addition, it can be easily adjusted to online environments, e.g.,

\footnotetext{
${ }^{1}$ Solution of one of the problems with 60 servers and 600 buffers took 131211 sec. Excluding this problem the average relative time becomes 484.46. Discretization to 1000 intervals is currently infeasible for larger MCQN problems.
} 
with rolling time horizon or model predictive control. Solution for a new period $\left[t_{0}, T+t_{0}\right]$ could be obtained by truncating the solution for time horizon $T$ at $t_{0}$ and then re-solving the problem starting from the truncated solution by increasing the time horizon from $T-t_{0}$ up to $T$ through its regular parametric line. During numerical experiments we found that the number of iterations of SCLP-simplex decreases exponentially with the growth of the time horizon that may be especially useful in these settings. On the other hand, in many cases the discretized LP model will need to be solved for the whole new time horizon from scratch since solutions of previous iterations may be infeasible for the new period.

There is a wide scope for further research and development of the continuous-time SCLP-simplex algorithms:

- Measure valued SCLP: while strong duality may fail in SCLP, formulation in the space of measures rather than the space of densities achieves strong duality, by allowing impulse controls at 0 and $T$, see [15], [16], [17].

- Piecewise constant data: can be solved similarly to [17].

- Continuous fractional programming: can be formulated as SCLP.

- Maximum flow over time with loses and arc delays: as formulated and discussed in [32], [33] is a challenging problem for which we may be able to characterize optimal solutions.

- Piecewise analytic objective and right hand side: these models were discussed in [8], [7], and it may be possible to define a simplex-type algorithm for them.

- General continuous linear programs: as formulated by Bellman [1] seem to be of a different nature than SCLP, and present a challenging area of future research.

\section{REFERENCES}

[1] R. Bellman, "Bottleneck problems and dynamic programming," Proceedings of the National Academy of Sciences of the United States of America, vol. 39, no. 9, p. 947, 1953.

[2] E. J. Anderson, "A new continuous model for job-shop scheduling," International J. of Systems Science, vol. 12, no. 12, pp. 1469-1475, 1981.

[3] G. Weiss, "A simplex based algorithm to solve separated continuous linear programs," Mathematical Programming, vol. 115, no. 1, pp. 151-198, 2008.

[4] C. E. Lemke and J. T. Howson, Jr, "Equilibrium points of bimatrix games," J. of the Society for industrial and Applied Mathematics, vol. 12, no. 2, pp. 413-423, 1964.

[5] E. J. Anderson and P. Nash, Linear programming in infinitedimensional spaces: theory and applications. John Wiley, 1987.

[6] M. C. Pullan, "An algorithm for a class of continuous linear programs," SIAM J. on Control and Optimization, vol. 31, no. 6, pp. 1558-1577, 1993.

[7] M. C. Pullan, "A duality theory for separated continuous linear programs," SIAM J. on Control and Optimization, vol. 34, no. 3, pp. 931-965, 1996.

[8] M. C. Pullan, "Forms of optimal solutions for separated continuous linear programs," SIAM J. on Control and Optimization, vol. 33, no. 6, pp. 1952-1977, 1995.

[9] M. C. Pullan, "Convergence of a general class of algorithms for separated continuous linear programs," SIAM J. on Optimization, vol. 10, no. 3, pp. 722-731, 2000.

[10] X. Luo and D. Bertsimas, "A new algorithm for stateconstrained separated continuous linear programs," SIAM J. on control and optimization, vol. 37, no. 1, pp. 177-210, 1998.
[11] L. Fleischer and J. Sethuraman, "Efficient algorithms for separated continuous linear programs: the multicommodity flow problem with holding costs and extensions," Math. of Oper. Research, vol. 30, no. 4, pp. 916-938, 2005.

[12] D. Bampou and D. Kuhn, "Polynomial approximations for continuous linear programs," SIAM J. on Optimization, vol. 22, no. 2 , pp. $628-648,2012$.

[13] X. Wang, S. Zhang, and D. D. Yao, "Separated continuous conic programming: strong duality and an approximation algorithm," SIAM J. on Control and Optimization, vol. 48, no. 4, pp. 2118$2138,2009$.

[14] A. Shapiro, "On duality theory of conic linear problems," in Semi-infinite programming, pp. 135-165, Springer, 2001.

[15] E. Shindin and G. Weiss, "Symmetric strong duality for a class of continuous linear programs with constant coefficients," SIAM J. on Optimization, vol. 24, no. 3, pp. 1102-1121, 2014.

[16] E. Shindin and G. Weiss, "Structure of solutions for continuous linear programs with constant coefficients," SIAM J. on Optimization, vol. 25, no. 3, pp. 1276-1297, 2015.

[17] E. Shindin and G. Weiss, "A simplex-type algorithm for continuous linear programs with constant coefficients," Mathematical Programming, pp. 1-45, 2018.

[18] P. Van Zant, Microchip Fabrication: A Practical Guide to Semiconductor Processing: A Practical Guide to Semiconductor Processing. McGraw Hill Professional, 2013.

[19] H. Chen, J. M. Harrison, A. Mandelbaum, A. Van Ackere, and L. M. Wein, "Empirical evaluation of a queueing network model for semiconductor wafer fabrication," Oper. Research, vol. 36, no. 2, pp. 202-215, 1988.

[20] L. M. Wein, "Scheduling semiconductor wafer fabrication," IEEE Transactions on semiconductor manufacturing, vol. 1, no. 3, pp. 115-130, 1988.

[21] P. Kumar, "Re-entrant lines," Queueing Systems, vol. 13, no. 13, pp. 87-110, 1993.

[22] D. Bertsimas, E. Nasrabadi, and I. C. Paschalidis, "Robust fluid processing networks," IEEE Transactions on Automatic Control, vol. 60, no. 3, pp. 715-728, 2014.

[23] J. M. Harrison, "Brownian models of queueing networks with heterogeneous customer populations," in Stochastic differential systems, stochastic control theory and applications, pp. 147-186, Springer, 1988.

[24] L. M. Wein, "Scheduling networks of queues: heavy traffic analysis of a multistation network with controllable inputs," Oper. Research, vol. 40, no. 3-supp.-2, pp. S312-S334, 1992.

[25] F. Kelly and C. Laws, "Dynamic routing in open queueing networks: Brownian models, cut constraints and resource pooling," Queueing systems, vol. 13, no. 1-3, pp. 47-86, 1993.

[26] J. G. Dai, "On positive harris recurrence of multiclass queueing networks: a unified approach via fluid limit models," The Annals of Applied Probability, pp. 49-77, 1995.

[27] M. Bramson, Stability of queueing networks. Springer, 2008.

[28] S. Meyn, Control techniques for complex networks. Cambridge University Press, 2008.

[29] Y. Nazarathy and G. Weiss, "Near optimal control of queueing networks over a finite time horizon," Annals of Oper. Research, vol. 170 , no. 1, p. 233, 2009.

[30] N. Gans, G. Koole, and A. Mandelbaum, "Telephone call centers: Tutorial, review and research prospects," Manufacturing and Services Operations Management, vol. 5, no. 2, pp. 79-141, 2003.

[31] A. Mandelbaum, P. Momčilović, and Y. Tseytlin, "On fair routing from emergency departments to hospital wards: Qed queues with heterogeneous servers," Management Science, vol. 58, no. 7, pp. 1273-1291, 2012.

[32] M. C. Pullan, "A study of general dynamic network programs with arc time-delays," SIAM J. on Optimization, vol. 7, no. 4, pp. 889-912, 1997.

[33] M. Groß and M. Skutella, "Generalized maximum flows over time," in International Workshop on Approximation and Online Algorithms, pp. 247-260, Springer, 2011. 